\title{
Alex Demirović
}

\section{Kritische Gesellschaftstheorie: Analyse der Kräfteverhältnisse oder Zeitdiagnose - mit einem Seitenblick auf die Beiträge von Slavoj Žižek}

In der marxistischen Gesellschaftstheorie haben sich zwei Weisen entwickelt, zwischen der allgemeinen Theorie und den konkreten gesellschaftlichen Situationen eine theoretische und praktische Beziehung herzustellen: das ist einmal die Zeitdiagnose in der Form von Ideologie- und Kulturkritik, zum anderen die Analyse von Kräfteverhältnissen. Beide haben verschiedene Gegenstände und Argumentationsweisen, ihr Bezug auf die Marxsche Theorie unterscheidet sich, und beide haben wohl auch deswegen zu einem unterschiedlichen Verständnis eben der Bedeutung der Kritik der politischen Ökonomie und ihrer gesellschaftstheoretischen Implikationen beigetragen.

Zeitdiagnose ist das Bemühen, zu einem genauen Verständnis der Gegenwart zu gelangen. Dieses Verständnis wird nicht oder nicht vorrangig in einer Analyse von ökonomischen Entwicklungen oder politischen Auseinandersetzungen gesucht. Vielmehr geht es darum, die konkrete Situation als eine solche der Individuen aus der Innensicht ihrer Subjektivität zu begreifen. Deswegen nehmen die Kultur und insbesondere die Ideologie in der Analyse ein großes Gewicht ein oder werden sogar zentral. Denn in den kulturellen Prozessen bilden sich der Sinn und die Bedeutungen und Deutungsmuster heraus, mit denen die Individuen sich selbst und ihre soziale Situation verstehen. Bedeutungen - seien sie symbolischer oder imaginärer Art - stellen eine interne Beziehungen der Individuen als Subjekte zu der gesellschaftlichen Konstellation her, in der sie leben. Auf diese Weise verspricht die Zeitdiagnose, ganz nahe an den Individuen, ihren Erfahrungen, ihren Bedürfnissen, ihrem Leiden und ihren Handlungsperspektiven zu sein. Gleichzeitig wird diese Sicht der Individuen intern mit gesamtgesellschaftlichen Entwicklungen in ein Verhältnis gesetzt, so dass die Erfahrungen der Einzelnen, der Sinn und die Bedeutungen, die sie sozialen Prozessen geben, in einem vermittelt-unmittelbaren Zusammenhang mit objektiven Vorgängen stehen. Im Unterschied dazu rückt die Analyse der Kräfteverhältnisse die ökonomische oder politische Lage der sozialen Klassen, ihres bestimmten Verhältnisses zueinander in der Ökonomie, im Verhältnis zum Staat und im Verhältnis zur kulturellen 
Organisation von Herrschaft ins Zentrum. Um das Verständnis von Zeitdiagnose näher zu erläutern, soll im Folgenden auf die Kritische Theorie Theodor W. Adornos zurückgegriffen werden. Denn seine Analysen werden, obwohl vor Jahrzehnten vorgelegt, nach wie vor für Diagnosen der aktuellen Situation genutzt (1). Eine theoretische Alternative zur Zeitdiagnostik findet sich in den Analysen von Kräfteverhältnissen und Konjunkturen sozialer Auseinandersetzungen. Um diesen Ansatz zu erläutern, werde ich auf Gramsci und Poulantzas eingehen (2). Vor diesem Hintergrund möchte ich mich schließlich mit dem Beitrag von Slavoj Žižek befassen, der innerhalb der globalen Linken maßgeblich zur marxistischen Theoriebildung und Zeitdiagnose beiträgt (3).

\section{Gesellschaftstheorie und kulturkritische Zeitdiagnose}

Eine der entscheidenden begrifflichen Instrumente, die Adorno für eine Zeitdiagnose fruchtbar macht, ist das Theorem des Äquivalententauschs. Damit ist ganz eng angelehnt an Marx’ Überlegungen in Band 1 des „Kapital“ gemeint, dass im Tauschvorgang in der Zirkulationssphäre ein spezifischer Prozess der Abstraktion vom Gebrauchscharakter der Gegenstände oder der konkreten Tätigkeiten stattfindet. Die Gegenstände ebenso wie die Individuen werden auf den einen, aber alles bestimmenden Aspekt eines Dritten, des Werts reduziert. Der Abstraktionsvorgang, der im Tausch vollzogen wird, ist kein bloß intellektueller Vorgang, sondern ereignet sich in der Wirklichkeit selbst, so dass die Individuen, ihre Tätigkeiten, ihre Produkte, ihre Bedürfnisse in diesem Vorgang des Gleichsetzens ihre Besonderheit verlieren. Deswegen ist mit dieser Reduktion schon ein wesentliches Moment von kulturkritischer Zeitdiagnose verbunden. Denn in solchen wertbestimmten Verhältnissen gilt keine Autonomie, alles ist Zweck für anderes. Die moderne Gesellschaft besteht, Adorno zufolge, vor allem aus Äquivalenzbeziehungen. Dies bedeutet, dass alle und alles gleich gesetzt wird, um es miteinander zu vergleichen. „In der Reduktion der Menschen auf Agenten und Träger des Warenaustauschs versteckt sich die Herrschaft von Menschen über Menschen.... Der totale Zusammenhang hat die Gestalt, daß alle dem Tauschgesetz sich unterwerfen müssen, wenn sie nicht zugrunde gehen wollen." (Adorno 1966, 14) Die Individuen, ihre Erfahrungen und Begriffe, ihre Handlungen und Tätigkeiten werden immer weiter der Gesellschaft unterworfen. Gesellschaft dehnt sich immer weiter aus, sie durchdringt alle Lebensbereiche mit dieser sie charakterisierenden Logik des Äquivalententauschs. Für Adorno ist ein wesentlicher Aspekt seiner Diagnose des Stands der kapitalistischen Vergesellschaftung, dass die bürgerliche Gesellschaft sich selbst die Einsicht in den wesentlichen Zusammenhang verschließt, indem sie leugnet, eine gesellschaftliche Totalität zu sein, die durch den 
Äquivalententausch hergestellt wird. Der Begriff der Gesellschaft wird in den Wissenschaften als metaphysisch verworfen, das Denken, intellektuelle Praxis, Theorie selbst werden mit einem Tabu belegt. Der funktionale Zusammenhang der Gesellschaft wird immer mehr zum System; sie wird immer umfassender und durchdringender, so dass ein weiteres Merkmal der Diagnose die Feststellung der Integration ist. „... und wenn man auf der andern Seite von 'der Gesellschaft' im modernen Sinn redet, hatte ich eigentlich bereits dieses im Auge, daß die Vergesellschaftung, also das Netz einfach der zwischen den Menschen gesponnenen gesellschaftlichen Beziehungen, sich immer enger und enger spannt." (Adorno 1968,73 ) Für Adorno ist dies ein wichtiger Befund. Denn Gesellschaft und Individuum sollten gleichsam symmetrisch durcheinander vermittelt sein. Kapitalistische Gesellschaftsverhältnisse, die ihrer vertragstheoretischen Ideologie nach geschaffen wurden, um allen Individuen Selbsterhaltung und Glück zu ermöglichen, konstituieren die Geltung des individuellen Anspruchs auf Gleichheit, Freiheit und auf vernünftige Gestaltung ihrer Lebensumstände. So kommt es überhaupt erst zur modernen Herausbildung der Spannung von Allgemeinem und Einzelnem. Die Entfaltung der Produktivkräfte würde diese Freiheit und Gleichheit als konkrete möglich machen. Doch sei ihre Verwirklichung verstellt. Im Namen der Selbsterhaltung wird der gesellschaftliche Reichtum genutzt, um Herrschaft aufrechtzuerhalten. Das ist ein wesentliches Bestimmungsmoment der kritischen Zeitdiagnose: dass der Moment der Befreiung versäumt wurde, dass die emanzipatorischen Denktraditionen, die sich in der Theorie von Marx symbolisch verdichten, nicht praktisch wurden, und bei aller Dynamik die kapitalistische Gesellschaft statisch wird und viele ihrer Vermittlungsmomente verliert. Dies trennt zwei große Phasen der kapitalistischen Entwicklung. Die kapitalistische Gesellschaft selbst nimmt die Form eines Objektivitätsüberhangs an, dem die Individuen unterworfen sind, der sich ihrer Möglichkeit der Erfahrung, der intellektuellen Durchdringung und schließlich der handelnden Veränderung entzieht. Die Entwicklung der Gesellschaft hin zu einem dichter und dichter integrierten System impliziert die Tendenz, dass die Menschen immer mehr dem System eingepasst und zu „mikrokosmischen Abbildern des Ganzen“ gemacht werden (ebd., 74). Integration heißt auch: der offene, revolutionäre Impuls, der von der Arbeiterklasse ausging, existiert nicht mehr, da sie mit den Mitteln der politischen Repression und der kulturindustriell-konsumistisch organisierten Lebensweise der bürgerlichen Gesellschaft eingeordnet worden ist, die versucht, den Widerspruch still zu stellen (vgl. Adorno 1964, 54). Da der Klassengegensatz, die Gegensätze von Reichtum und Armut, Macht und Ohnmacht fortexistieren, impliziert ihre Leugnung ein enormes Maß an direkter und symbolischer Gewalt; das Verdrängte bringt sich in einer Vielzahl von neurotischen Symptomen zur Geltung: fortdauernder Antisemitismus, Ablehnung von objektiver Vernunft und 
anspruchsvoller Theorie, Irrationalität, Ressentiment, antidemokratische Haltungen ... Individuen werden auf manipulierbare Reflexbündeln reduziert, die gleichsam von den Herrschaftszentralen durch eine Reihe von politischen und kulturindustriellen Mechanismen in den Dienst genommen werden. Den zum Konformismus angehaltenen Individuen wird die Möglichkeit von Bewusstsein, Reflexion und Erfahrung genommen. Sie orientieren sich an der Macht des Faktischen. Dass es so ist, wie es ist, wird selbst zur Ideologie - Ideologie durchläuft also in der kapitalistischen Gesellschaft einen Funktionswandel: war sie, getrennt von der Sphäre der Arbeit und der Verwertung, lange ein Bereich der Bedeutungen, in denen das Falsche und das Wahre miteinander verbunden waren, so werden unter dem Spätkapitalismus die kulturindustriell reorganisierten gesellschaftlichen Verhältnisse selbst zur Ideologie, die unmittelbare Macht des Faktischen eignet sich die Sphäre der Bedeutung an, so dass diese ihre kritische Distanz verliert. Diese Veränderungen, so kann Adorno verstanden werden, verstehen auch viele marxistische Kritiker nicht, weil sie affirmativ sind. Denn im Namen des Materialismus advozierten sie die objektiv mächtigsten Tendenzen (Adorno 1951, 48); diesen gegenüber seien auch sie konformistisch, anstatt den Materialismus selbst kritisch infrage zu stellen. Auch sie ließen sich von solchen Tendenzen vorgeben, worüber nachgedacht, was als relevant angesehen, für objektiv gehalten wird. Am Wahn der Größe und Kultus des Wichtigen offenbare sich etwas Unfreies. „Das bedeutet nicht, daß die Hierarchie der Wichtigkeiten zu ignorieren sei. Wie ihre Banausie die des Systems widerspiegelt, so ist sie gesättigt mit all seiner Gewalt und Stringenz. Jedoch der Gedanke sollte sie nicht repetieren, sondern im Nachvollzug auflösen. Die Aufteilung der Welt in Haupt- und Nebensachen, die schon immer dazu gedient hat, die Schlüsselphänomene des äußersten gesellschaftlichen Unrechts als bloße Ausnahmen zu neutralisieren, ist soweit zu befolgen, daß sie ihrer eigenen Unwahrheit überführt wird. Sie, die alles zu Objekten macht, muß selber zum Objekt des Gedankens werden, anstatt ihn zu steuern. Die großen Themen werden dabei auch vorkommen, aber kaum im traditionellen Sinn $\gg$ thematisch «, sondern gebrochen und exzentrisch." (Adorno 1951, 140) Die Zeitdiagnose der kritischen Gesellschaftstheorie zielt darauf, die Wertigkeiten umzukehren, die Gegenstände zu konstituieren, sie bemüht sich um eine Perspektive, in der die Individuen begreifen, dass sie selbst durch ihr Handeln, durch ihre Vernunft, durch ihre Lebensformen die Gesellschaft vermitteln. „Ich würde nicht zögern, die Idee einer dialektischen Lehre von der Gesellschaft als etwas, ja, wie die Wiederherstellung oder die Anstrengung zu definieren, die Erfahrung, die uns eigentlich von dem sozialen System selbst ebenso wie von den Regeln der Wissenschaft versagt wird, wiederherzustellen. Man könnte sagen, das, was ich hier versuche Ihnen zu entfalten, ist so etwas wie die Grundsätze einer Rebellion der Erfahrung gegen den Empirismus." (Adorno 1968, 91) 
Zeitdiagnostische Gesellschaftstheorie hat für Adorno eine doppelte Aufgabe. Einmal folgt sie kritisch dem Prozess, in dem die Logik des Äquivalententauschs fortschreitet, Emanzipation verhindert und damit die Individuen und ihr Leben der Vergesellschaftung bis hin zur totalen Vergesellschaftung und Verwaltung unterwirft, die gesellschaftlichen Widersprüche unterdrückt, Besonderheiten eliminiert und die individuelle Autonomie beseitigt. Was also geschieht den Individuen und ihren Verhältnissen, wenn sie in einer Phase leben, in der die Verwirklichung der Aufklärung mit Macht blockiert wird, gleichzeitig aber weiterhin Emanzipation, Freiheit, Gleichheit und Vernunft versprochen wird? Welche Erfahrungen machen sie, wenn sie in einem solchen Widerspruch leben müssen? Wie deuten sie ihre Situation, in welcher Beziehung stehen sie zum Allgemeinen? Adorno gibt der Erfahrung der Individuen, ihrer Binnensicht eine zentrale Bedeutung in seiner Zeitdiagnose. Zum zweiten hält die Zeitdiagnose aber auch fest, in welchem Maße Residuen der Freiheit immer noch Bestand haben und sich ungleichzeitig der Subsumtion unter den Tausch, damit der Heteronomie entziehen und den Raum für kritisches Denken und Handeln gewähren.

Die Zeitdiagnose hat theoretisch-methodische Schwächen, von denen Adorno selbst einige durchaus gesehen und verteidigt hat: a) Sie hat eine implizite Neigung, frühere Zustände als die besseren gegenüber den gegenwärtigen zu stilisieren und dadurch eine Atmosphäre des Verlusts, der Nostalgie zu erzeugen. b) Sie hat die Tendenz zur Übertreibung. Das Verhältnis der empirischen Bedeutung einzelner Phänomene zur Gesellschaftstheorie bleibt oftmals in der Schwebe. Bemerkenswert ist in diesem Zusammenhang, dass Adorno nie versucht hat, seine Überlegungen zur Kulturindustrie kapitalismustheoretisch-empirisch weiter auszuarbeiten. Es müssten jedenfalls viele zeitdiagnostischen Einschätzungen in diesem Sinn aus heutiger Sicht eher in Frage gestellt werden. c) In der Diagnose drängen sich die Dynamiken, die die zunehmende Integration bewirken, vor jene Prozesse, in denen sich Individuen dem Konformismus entziehen. Widerspruchskonstellationen werden also einseitig dargestellt. d) Die Fallhöhe ist in der Analyse groß: auf der einen Seite die eher sehr allgemeinen Überlegungen zum Äquivalententausch und dem Kapital im Allgemeinen, auf der anderen Seite die Kultur in Gestalt von Kunstwerken und empirisch feststellbaren Meinungen und Einstellungen. Dabei werden kategorial verschiedene Ebenen in eins gesetzt: gesellschaftstheoretische Begriffe, die der Analyse dienen, werden auch als zeitdiagnostischer Befund präsentiert. e) Aus guten Gründen will Adorno Konkretismus und Personalisierung vermeiden. Denn auch das ist ein Moment seiner Gesellschaftstheorie und Zeitdiagnose: dass immer abstraktere Herrschaftsbeziehungen, dass blinde Herrschaft des Tauschwerts oder der gesellschaftlichen Arbeitsteilung konkretistisch an bestimmten Individuen festgemacht werden. Doch spricht Adorno auch von den Mächtigen, von den Herrschenden und ihren 
Funktionären. Wenn er ausführt, dass die Marxsche Theorie Herrschaft aus dem Tauschverhältnis ableite, so meint er, demgegenüber die häretische Ansicht zu äußern, dass der entscheidende Tauschakt, der der lebendigen Arbeit gegen den Lohn, in Wirklichkeit bereits das Klassenverhältnis voraussetzt und durch dieses entscheidend gemodelt worden sei (vgl. Adorno 1964, 97). Mit dieser Bemerkung weist Adorno auf eine Analyse von Herrschaftspraktiken und Kräftekonstellationen hin, die in seinen eigenen Studien jedoch kaum explizit unternommen wird.

\section{Gesellschaftstheorie und die Analyse von Kräfteverhältnissen}

Gesellschaftstheorie, die auf die Analyse einer konkreten Konjunktur von Klassenkämpfen zielt, um es mit Nicos Poulantzas zu sagen, setzt genau an diesem Punkt der Rolle von Herrschaft an, der von Adorno zwar angesprochen, aber nicht weiter diskutiert wird. Wie im Fall von Adornos Zeitdiagnostik nimmt sie an, dass die bürgerliche Gesellschaft nicht an ihren Rändern, sondern von innen durch grundlegende Widersprüche bestimmt ist. Klassen bilden maßgebliche Kräfte, die sich in besonderen Verhältnissen konstituieren und in ihren Kämpfen reproduzieren und ständig verändern. Diese Klassen erzeugen in ihren Auseinandersetzungen sich selbst ebenso wie die antagonistischen Verhältnisse, die sie zwingen, wiederum weiter zu kämpfen. In diesem Prozess ändern sich ständig alle Verhältnisse und damit auch die konkrete Gestalt der gesellschaftlichen Akteure, ihrer Praktiken und Identitäten. Es ist eine spezifische Dialektik von Identität und Nichtidentität der sozialen Akteure: die Klassen bleiben diese bestimmten Klassen, obwohl sie sich verändern. Aus dieser Veränderung kann im Rahmen der Zeitdiagnose Adornos die Frage entstehen, ob sich nicht, wenn es auf der Ebene der Erscheinung keine Klassen mehr gibt, auch das Wesen der kapitalistischen Verhältnisse ändert. Adorno hat dies verneint und hat prinzipiell am Klassencharakter des Spätkapitalismus festgehalten. Im Fall der Analyse von Kräfteverhältnissen wird demgegenüber angenommen, dass diese Veränderungen sich aus der Relation der Klassen selbst ergeben, die Klassen also ihren Charakter und damit auch die Form ihrer Auseinandersetzung verändert haben. Die bürgerliche Gesellschaft wird als ein die Ökonomie, den Staat, die Kultur und die Individuen umfassender Organismus begriffen, der sich permanent selbst revolutioniert. So verstanden, wären aber alle Katzen grau; es geht konkret um die Bestimmung von Kräftekonstellationen. Diese Bestimmung umfasst im Prinzip die Gesamtheit aller Lebensverhältnisse. Gramsci (1996, 1556ff) unterscheidet mehrere Ebenen von Kräfteverhältnissen:

a) Ein eng an die Struktur gebundenes, das objektiv und vom Willen der Menschen unabhängig ist: Anzahl der Unternehmen und ihrer Beschäftigten oder 
Städte und ihrer Einwohner. Gramscis Überlegungen weisen daraufhin, dass sich anschließend an Marx’ Ausführungen im „Kapital“ der Begriff des Kräfteverhältnisses zwischen den Klassen schon auf der Ebene der Ökonomie sehr weit fassen lässt. Es geht konkret darum, wie sich die Aneignung von Mehrarbeit vollzieht, allein die Analyse des Vorgangs des Äquivalententauschs wird als zu unspezifisch angesehen. Zunächst einmal ist ein Merkmal des Kräfteverhältnisses, ob es zu gewaltsamer Enteignung kommt (Raub von Land oder genetischen Ressourcen, Zwangsarbeit, Schuldknechtschaft) oder sich als verstetigte Aneignung in der formbestimmten Ökonomie vollzieht. Die Aneignung des lebendigen Arbeitsvermögens findet dann in spezifischen Formen unter bestimmten politischen Bedingungen statt: Betriebsweise, Lohnform, Arbeitsprozesse (Arbeitszeit und -belastung, Arbeitsschutz, Pausen), Organisation der gesellschaftlichen Kooperation und technologischen Verhältnisse, Trennung von körperlicher und geistiger Arbeit, von Ausführung und Kommando über die Arbeit. Das Verhältnis von Branchen und Unternehmen und die Auseinandersetzung darum, welche Anteile sie aus dem Gesamtprofit des Gesamtarbeiters ziehen können.

b) Das politische Kräfteverhältnis, das Gramsci in mehrere Momente unterscheidet: erstens das korporativ-ökonomische Moment, das die Kapitaleigentümer eines Zweigs zu einer Fraktion miteinander verbindet. Zweitens das Moment des Bewusstseins der Interessensolidarität als ökonomische Klasse. Auf der ökonomischen Ebene kommt es bereits - worauf auch Franz Neumann hingewiesen hat zu Formen der herrschaftlichen Organisation: Wirtschaftsverbände auf der Seite der Kapitaleigentümer, Managementausbildung, Beratungseinrichtungen für den Umgang mit Lohnabhängigen, regelmäßige Treffen zwischen Unternehmern, Managern und Wirtschaftsführern (Weltwirtschaftsforum, European Round Table of Industrialists). Auf der Gegenseite bilden sich Formen der ökonomischpolitischen Organisation der Lohnabhängigen und ihrer alltäglichen Praktiken des Widerstands, die vom gewerkschaftlichen Engagement und Dienst nach Vorschrift über die Sabotage von Arbeitsabläufen bis zum offenen Arbeitskampf reichen kann. Ein drittes Moment ist das der Verallgemeinerung der Interessen derjenigen, die über den Produktionsapparat verfügen, auf andere, untergeordnete Gruppen. Dieses Moment markiert den Übergang von der Sphäre der Basis zu den Überbauten, also den Vorgang, dass eine Gruppe oder eine Allianz sich verallgemeinert, Hegemonie erlangt und eine politische, intellektuelle und moralische Einheit, also Universalität und die universelle Geltung einer Lebensweise herstellt. Gramsci hat mit einer solchen Konzeption der Zivilgesellschaft als Bereich der Ausübung von Hegemonie den Begriff des Klassenkampfs auf den gesamten Bereich der Organisation der Kultur ausgedehnt. Dies umfasst die kollektiven Gewohnheiten im Alltag, also Essgewohnheiten, Sexualpraktiken, Familienformen, religiöse Überzeugungen, Leseverhalten, Alltagswissen, das 
Verhältnis zu Wissenschaften und Kunst. Alle diese Praktiken sollen als ebenso viele mögliche Kräfteverhältnisse zwischen den sozialen Klassen dechiffriert werden. Doch die allgemein-begriffliche Fassung der Kräfteverhältnisse selbst ist von deren konkreter Analyse selbst zu unterscheiden.

Politisch im offen staatlichen und rechtlichen Sinn sind solche Kräfteverhältnisse, wo in alltäglichen Auseinandersetzungen darum gerungen wird, soziale Verhältnisse entlang der Unterscheidung von partikular-allgemein zu thematisieren oder zu dethematisieren. Gelingt es, Fakten, Praktiken, Verhältnisse derart zu politisieren, setzen sich die Machtauseinandersetzungen auf höherer Stufe als formeller politischer Konflikt um staatliche Macht fort. Das Thema polarisiert und trägt zur Bildung von Parteien bei, die um die Festlegung der gesamtgesellschaftlich bindenden Entscheidungen kämpfen. Dabei handelt es sich um Parteiungen auf gesamtgesellschaftlicher Ebene, die das kollektive Leben von sozialen Gruppen insgesamt umfassen. Sie sind zu unterscheiden von dem „unmittelbaren Moment" des Kräfteverhältnisses. Damit meint Gramsci die Verhältnisse der in verschiedenen Parteiformen (Zeitungsleser, Parlamentswahlen, Mitgliedschaften in Parteien und Gewerkschaften) organisierten politischen Kräfte.

Nicos Poulantzas $(1974,308 \mathrm{ff})$ hat diese Ebene von politischen Kräfteverhältnissen als Regimeform bezeichnet und von der Staatsform unterschieden. Diese bezeichnet eine besondere Gliederung von Ökonomie und Staat, also die spezifische Art und Weise, wie der Staat die herrschenden Klassen und ihre Fraktionen im Verhältnis zu den beherrschten Klassen organisiert und eine spezifische relative Autonomie gegenüber dem Machtblock gewinnt. Die Gliederung und relative Autonomie der verschiedenen gesellschaftlichen Handlungslogiken sind demnach selbst noch Ergebnis von Klassenkampf und in ihrer Spezifität Merkmal einer Konjunktur. Diese besondere Modalität der Staatsform bringt sich zur Geltung in der Dominanz eines Staatsapparats, der die Staatsapparate in ihrer Gesamtheit vereinheitlicht und in der Tendenz eine kohärente Politik gewährleistet. Charakteristisch für das Kräfteverhältnis ist für Gramsci ebenso wie für Poulantzas also das Verhältnis der verschiedenen Gruppen der herrschenden Klassen zueinander und ihr Verhältnis gegenüber den beherrschten Klassen, also kleinbürgerliche Klasse und ArbeiterInnenklasse, sowie die unterschiedlichen Allianzen, die sich jeweils ergeben. Die Kräfteverhältnisse, die die Form bestimmter Konstellation von Allianzen und Polarisierungen annehmen, haben unterschiedliche zeitliche Rhythmen. Auf der Ebene der Staatsformen bestehen sie unter Umständen über mehrere Jahrzehnte, während sie auf der Ebene der Regimeform schnell wechseln können. Eine wesentliche Erweiterung, die Poulantzas gegenüber Gramsci vornimmt, ist die Bestimmung der besonderen Rolle einzelner Staatsapparate innerhalb einer komplexen Kräftekonstellation. Nicht zuletzt damit lassen sich auch Formen des Ausnahmestaats unterscheiden, wenn 
nämlich die Gesamtheit der Staatsapparate durch solche Apparate wie Polizei oder Militär vereinheitlicht wird.

c) Schließlich ist noch das militärische Kräfteverhältnis zu nennen, das von Gramsci unterschieden wird in ein militärisches und ein militärisch-politisches Verhältnis. Als Beispiel für letzteres nennt er die Unterdrückung einer Nation, die ihre staatliche Unabhängigkeit zu erlangen sucht.

Ein besonderer Aspekt der Analyse von Kräfteverhältnissen ist die Analyse von Ideologie. Adorno hat die historische Veränderung der Ideologie zeitdiagnostisch begriffen: in der bürgerlichen Gesellschaft ändert sich mit der Herausbildung der Kulturindustrie das Verhältnis von Basis und Überbau und damit die Rolle von Bedeutungsprozessen und Vernunft. Die bürgerliche Gesellschaft verschließt sich zunehmend ihrem Begriff. Anders verhält es sich im Fall der von Althusser (2010) partiell an Gramsci anschließenden Überlegungen, Ideologie als eine materielle Praxis zu begreifen, die in spezifischen Staatsapparaten wie Kirche, Schule, Hochschulen oder Parteien organisiert ist. Auch in diesem Fall sollen sich besondere Kräfteverhältnisse bestimmen lassen; es handelt sich um objektive Bedeutungspraktiken. Diese Kräfteverhältnisse sind auch ausschlaggebend dafür, dass sich die „Subjektivität“ und die Identität des Individuums herausbilden, das sich imaginär im Verhältnis zu sich selbst als willens- und bewusstseinsbegabt repräsentiert. Die eindrücklichsten Arbeiten dazu hat Michel Foucault beigetragen, ohne sich dann noch weiter auf die Begriffe „Ideologie“ oder „ideologischer Staatsapparat" zu beziehen.

Entscheidend ist in diesem Verständnis, dass die Verhältnisse zwischen Klassen und ihre Kämpfe den gesellschaftlichen Gewohnheiten und Institutionen immanent sind. Sie treten ihnen nicht als ein Phänomen zusätzlich noch einmal von außen hinzu, sondern vollziehen sich in den materiellen Verhältnissen von Ökonomie, Politik und Kultur. In diesem Sinn ist Gramscis Satz zu verstehen, wonach „das staatliche Leben als ein ständiges Sich-Bilden und Überwunden-Werden instabiler Gleichgewichte zwischen den Interessen der grundlegenden Gruppe und denen der untergeordneten Gruppen aufgefaßt wird, Gleichgewichte, in denen die Interessen der grundlegenden Gruppe überwiegen, aber nur bis zu einem gewissen Punkt, also nicht bis zum nackten korporativ-ökonomischen Interesse“ (Gramsci 1996, 1561) Die permanente Selbstrevolutionierung der bürgerlichen Gesellschaft wird demnach nicht als ein irgendwie linear gerichteter, gar als ein Rationalisierungs- und Modernisierungsprozess verstanden. Vielmehr setzen die sozialen Klassen ihre Auseinandersetzung in immer neuen Konstellationen fort, bis unter der Hegemonie einer neuen Klasse die Gesamtheit aller Verhältnisse in einem langwierigen Prozess transformiert werden und allmählich dann auch jene Verhältnisse beseitigt werden können, in denen sich der Klassenkonflikt und die Trennung von ausführender und konzeptiver Arbeit reproduziert. 
Die Stärke einer Analyse der Kräfteverhältnisse besteht darin, die Ebene der theoretischen Begriffe und die der mit ihnen durchgeführten Analyse sorgfältig zu trennen. Es wird die konkrete Konjunktur in einer Vielzahl von gesellschaftlichen Verhältnissen in den Blick genommen und die Akteure und ihre Praktiken untersucht. Ein zentraler Gesichtspunkt der Analyse ist, wie sich eine partikulare gesellschaftliche Kraft in konkreten Praktiken ausdehnt und universalisiert und damit den gesamtgesellschaftlichen Zusammenhang organisiert. Auf zwei Defizite soll hingewiesen werden. Zum einen kommen die Innensicht von Individuen, ihre Erfahrungen, die psychischen Dynamiken nicht in den Blick. Vielleicht bedeutsamer noch ist, dass ein gewisses geschichtsphilosophisches Moment fehlt, also die Bestimmung des historischen Bezugspunkts, von dem aus der historische Stand der Emanzipation, das Gewicht der Theorie, die Bedeutung der Akteure ermessen werden kann. Das mag seine Vorteile haben, weil Nüchternheit hinsichtlich der konkreten gesellschaftlichen Entwicklung besteht; gleichzeitig jedoch kann die Theorie nicht oder wenig auf die Stimmung von Individuen reagieren oder zu deren Formierung beitragen. Für die Weiterentwicklung der materialistischen Gesellschaftstheorie erscheint es sinnvoll, diese beiden komplementären Ansätze der Zeitdiagnose und der Untersuchung von Kräfteverhältnissen in einer integralen Theorie zusammenzuführen; sinnvoll deswegen, weil damit Defizite vermieden und Vorwürfe, materialistische Ansätze seien funktionalistisch und könnten die Handlungsperspektive nicht einnehmen, unterlaufen werden könnten. Es sollte auch möglich sein, weil sich in beiden Ansätzen jeweils auch schon Elemente des jeweils anderen finden.

\section{3 Žižeks Ideologiekritik des Neoliberalismus}

Im Folgenden will ich auf einige Überlegungen Slavoj Žižeks eingehen. Seine Texte legen durch den Bezug auf Marxismus und Psychoanalyse nahe, dass sie das Potential haben beide Typen der Analyse, theoretische Analyse der Kräfteverhältnisse und Zeitdiagnose, zusammenzubringen. Die Aufgabe der Linken ist aus seiner Sicht, die Position der Mächtigen mit geduldiger Ideologiekritik zu unterminieren (Žižek 2011, 11). Damit verleiht er dem zeitdiagnostischen Moment der Analyse eine neue starke Bedeutung. Dies gibt die Wahl der Titel mancher seiner Bücher zu verstehen: Willkommen in interessanten Zeiten! oder Die Revolution steht bevor. Auch seine zahlreichen Rückgriffe auf kulturwissenschaftliche Erkenntnisse, auf Musik, Film, Fernsehen oder Literatur legen dies nahe. Das Projekt von Žižek ist eine psychoanalytisch informierte Ideologiekritik, die auf subjektive Erfahrungen rekurriert und den Positivismus der affirmativ gewordenen Cultural Studies zurückweist - aber es greift auch auf Elemente 
einer Analyse von Kräfteverhältnissen zurück und will sie ihrerseits zeitdiagnostisch fruchtbar machen. Dass Žižek mit seinen Analysen etwas trifft, zeigt die Resonanz auf seine zahlreichen Publikationen und seine Internetpräsenz ebenso wie die großen TeilnehmerInnenzahlen bei öffentlichen Veranstaltungen mit ihm. Er gehört zu einem neuen Typus von kritischen Intellektuellen, die global wirken (Foreign Policy zählte ihn 2012 an Stelle 92 zu den 100 globalen Denkern). Zu diesem Typus kritischer Intellektueller gehören Antonio Negri und Michael Hardt, Alain Badiou, Jacques Rancière, Judith Butler, Gayatri Spivak, Wendy Brown, Ernesto Laclau, Chantal Mouffe oder Etienne Balibar. Sie alle sind mit sozialen Protesten und sozialen Bewegungen der vergangenen Jahrzehnte verbunden, die sich gegen kapitalistische Verhältnisse und ihre gegenwärtige neoliberale Form wenden. Diese global wirksamen kritischen Intellektuellen mediatisieren in starkem Maße die kritischen Diskussionen auf nationalstaatlicher Ebene, die selbst aber wiederum von diesem globalen Prozess der Theoriebildung getrennt bleiben, so dass sich mehrere Ebenen linker, emanzipatorischer Diskussion bilden, zwischen denen es nur an wenigen Stellen zum Austausch kommt. ${ }^{1}$ Theoretisch stützen sich diese Intellektuellen auf die sog. poststrukturalistischen Theorien von Althusser, Foucault, Deleuze oder Lacan. Žižek ist in diesem Feld derjenige, der in seinen politischen und Kulturanalysen teilweise harte Kritik an dieser Tradition sowie den anderen kritischen Intellektuellen und Strömungen äußert. „Also, I really hate all of this politically correct, cultural studies bullshit. If you mention the phrase „postcolonialism," I say, „Fuck it!“ Postcolonialism is the invention of some rich guys from India who saw that they could make a good career in top Western universities by playing on the guilt of white liberals." In diesem Sinn einer kritischen Haltung stellt er deutliche Differenzen heraus. Das ist weder selbstverständlich noch bedeutungslos. Denn im Unterschied zu vielen anderen der erwähnten global wirksamen Intellektuellen plädiert er für die Fortgeltung der Theorie von Marx und für eine an ihn anschließende Gesellschaftsanalyse. Es lässt sich wohl sagen, dass er aufgrund seiner globalen Wirksamkeit de facto einer der bedeutsamen öffentlichen marxistischen Stimmen in Deutschland ist und etwas sagbar macht. So wirbt der S. Fischer Verlag damit, dass Žižek die Widersprüche

1 So konnte im Juni 2010 an der Volksbühne in Berlin eine dreitägige Veranstaltung u.a. mit Vorträgen von Žižek und Badiou vor knapp 1000 TeilnehmerInnen zur Idee des Kommunismus durchgeführt werden, ohne dass dies einen näheren Bezug zu Debatten in der bundesdeutschen Linken gehabt hätte. Vgl. Douzinas/Žižek 2012 und Badiou/Žižek 2012. Ähnlich galt dies auch für die von Žižek organisierte Tagung „Gibt es eine Politik der Wahrheit - nach Lenin?" am Kulturwissenschaftlichen Institut in Essen im Februar 2001 (http://www.freitag.de/autoren/der-freitag/die-leninistische-geste). Vgl. Žižek 2002.

2 http://www.salon.com/2012/12/29/slavoj_zizek_i_am_not_the_worlds_hippest_philosopher/ 
der gegenwärtigen politischen Lage freilege und danach frage, „wie wir das System bekämpfen können, ohne zu seinem Funktionieren beizutragen“"(Umschlagstext zu Žižek 2013). So wie mit Blick auf diese globalen Intellektuellen und ihre Wirkung die Meinungen innerhalb der Linken weit auseinandergehen, gilt dies auch für Žižek selbst. Für die einen mag er ein cooler und einflussreicher Intellektueller sein - „a cult icon and spiritual guide for Europe’s lethargic left“ - , den anderen gilt er als wahnhaft, als Clown, als „Salonstalinist“ oder als „Antisemit“.3

Žižeks Theoriebildung vollzieht sich patchworkartig. In den Büchern der vergangenen Jahre werden ausführlich die politischen und sozialen Gegebenheiten der kapitalistischen Gesellschaften geschildert: Europa, USA, China, Türkei, es wird auf die Bewegungen des arabischen Frühlings eingegangen oder die Lage im Nahen Osten. Diese Ausführungen sind durchdrungen mit theoretischen Ausführungen: gelegentliche Andeutungen zur Französischen Revolution, zu Badiou oder Walter Benjamin oder der politischen Theorie, die dann an anderer Stelle wieder aufgegriffen und manchmal umfänglich ausgeführt werden. Es lassen sich also oftmals weniger eine These oder Theorem als eine Reihe von Motiven feststellen, die sich assoziativ entfalten. Manche Kritiker halten aufgrund dieser essayartigen Schreibweise Žižeks Texte für philosophisch leer. ${ }^{4}$ Dabei wird jedoch seine Bemühung außer Betracht gelassen, durch Kritik an relevanten Ideologemen Selbstverständlichkeiten in der Linken in Frage zu stellen - „einen Glaubenssprung riskieren“ (Žižek 2009, 27) - und die Begriffe der linken und marxistischen Tradition wieder zu gewinnen. Wahrheit sei zugänglich nur, wenn man einen Standpunkt einnehme, doch werde sie dadurch nicht weniger universell - sein eigener Standpunkt sei natürlich der des Kommunismus, dieser müsse wieder ins Spiel gebracht werden; die Botschaft laute: man dürfe wieder getreu der kommunistischen Idee handeln (vgl. Žižek 2009, 13; Žižek 2011, 8). Žižek lehnt es als Zumutung ab, dass die Linke sich aufgrund des Einschnitts von 1989/1990 neu erfinden solle, vielmehr solle sie von Anfang an beginnen, den Anfang wiederholen. In der Wiederholung selbst wird auch die Notwendigkeit der historischen Entwicklung neu konstituiert. Die Wiederholung selbst ist jedoch nur möglich, wenn der heutige Kapitalismus Antagonismen beinhaltet, „die stark genug sind, um seine unendliche Reproduktion zu verhindern. Das sind: die ökologische Katastrophe, die Unangemessenheit der Idee des Privateigentums für das sog. geistige Eigentum, die Biogenetik sowie neue Formen der Apartheid (Žižek 2011, 97, 300). Interessanterweise spielen Arbeitsverhältnisse,

3 http://www.salon.com/2012/12/29/slavoj_zizek_i_am_not_the_worlds_hippest_philosopher/; http://www.welt.de/print/die_welt/kultur/article13371383/Eheberatung-mitHegel.html

4 http://de.wikipedia.org/wiki/Slavoj_\%C5\%BDi\%C5\%BEek 
Formen der Ausbeutung, die konkrete Existenz von Lohnarbeitenden Herrschaftsverhältnisse, die globalen Machtverhältnisse in dieser Aufzählung keine Rolle.

Eine der wichtigen und immer wiederkehrenden Motive in Žižeks Texten ist sein Plädoyer dafür, „die Natur des heutigen Kapitalismus zu begreifen“ (Žižek 2009, 196). Dessen Merkmale sind ein langfristiger Trend der Verlagerung von Profit auf Rendite (Rendite für privatisiertes kollektives Wissen und natürliche Ressourcen); die strukturelle Rolle der Arbeitslosigkeit und schließlich der Aufstieg einer neuen Klasse von Angestellten: Manager, Experten, Ärzte, Rechtsanwälte, Journalisten oder Intellektuelle (vgl. Žižek 2013, 15ff.). Es wiederholt sich hier, dass der Kapitalismus durch einige vage ökonomische Merkmale bestimmt wird, was nahe legt, dass die Analysen Žižeks einen diesem äußerlichen Gegenstand betreffen. Er argumentiert, dass eine solche theoretische Bemühung nur gelingen könne, wenn man bereit ist, die Alternative zu denken und zu begehren. „Die Naturalisierung des globalen Kapitalismus hat ein noch nie dagewesenes Ausmaß erreicht. Mittlerweile gibt es kaum noch jemanden, der utopische Träume über mögliche Alternativen auch nur zu träumen wagt." (Žižek 2011, 289) Eine Schlüsselfrage sei deswegen, wie die Wirklichkeit symbolisiert wird. Die Gefahr bestünde darin, dass sich eine Erzählung durchsetzt, „die uns nicht aufweckt, sondern die es ermöglicht weiterzuträumen" (Žižek 2009, 23). Der Liberalismus ist genau darum bemüht. Er erzeugt eine Traumwirklichkeit - und es kann Žižeks theoretische Arbeit vielleicht so verstanden werden, dass sie sich gerade dem Liberalismus und der von ihm erzeugten Wirkungen mit einem eigenartigen ontologischen Status zuwendet: ein Traum, der doch ganz viele Folgen hat.

Der Liberalismus zielt darauf, die Gesellschaft zu normalisieren und Markt und Demokratie als die natürliche Form der Gesellschaft erscheinen zu lassen. Das ist sogar noch dann möglich, wenn der Ausnahmezustand eingeführt oder Wirtschaftskrise als eine schon längst bewältigte Rezession dargestellt wird (vgl. Žižek 2011,252; 2009, 9ff). Ein Merkmal des Liberalismus ist seine Ambiguität. Er verwickelt in ein Dilemma: man weiß nicht, was der wahre Liberalismus ist, denn seine Formen treten als Gegenteil ihrer jeweils anderen Form auf, als Markt- oder als politischer Liberalismus und multikulturalistischer Humanismus. Der politische Liberalismus lehnt den Eingriff in die bestehenden Lebensweisen ebenso wie Utopien ab, weil dies autoritäre Folgen haben könnte. Faktisch aber, so Žižek, würde dies nur zur Durchsetzung der marktliberalen Utopie führen. Der Neoliberalismus erscheine als Zeichen eines neuen Zeitalters, das die utopischen Projekte hinter sich gelassen habe, die vermeintlich für die totalitären Schrecken verantwortlich gewesen wären. Postideologisch bildeten sich alle ein, nicht mehr wirklich an ,unsere Ideologie zu glauben - trotz dieser imaginären Distanz üben wir sie weiterhin aus“ (Žižek 2011, 8) Der Markt selbst sei eine Utopie, die enorm viel Glaube, Vertrauen und Gewalt erfordere, damit sein Funktionieren 
aufrechterhalten werden kann (vgl. Žižek 2009, 52, 11). Der Liberalismus als herrschende Ideologie konstruiert einen falschen Widerspruch: liberale Demokratie, Markt, Freiheit auf der einen Seite, Fundamentalismus, Antimodernismus, Terrorismus, Totalitarismus, Islamfaschismus auf der anderen Seite. Er bietet allein die Alternative, dass man entweder totalitär und autoritär oder tolerant und offen ist. Es ist eine bestimmte Art von Unentschiedenheit und Permissivität, die Wendy Brown in ihrer Analyse des liberalen Toleranzdiskurses beobachtet hat. Gerade das, was eigentlich toleriert werden müsste, das Andere am Anderen, wird neutralisiert - die Anderen müssen auf ihre Überzeugungen und Lebensformen dort, wo sie verbindlich werden, verzichten und sich einfügen (vgl. Brown 2000, 257ff). Permissivität bedeute gerade das: den Rahmen des Erlaubten für die Einzelnen zu erweitern, ohne ihnen Macht zu geben (vgl. Žižek 2011, 267). In seiner Auseinandersetzung mit dem Islam argumentiert Žižek, dass dieser Traditionen kenne, die das Ziel einer auf Vernunft und Gleichheit beruhenden Gesellschaft verfolgt hätten. Der Westen sei heuchlerisch, denn angesichts der Auflehnung des ägyptischen Volkes gegen Mubarak im Namen von Freiheit und Gerechtigkeit habe Tony Blair den stabilen Wechsel gefordert. „Nein, wir brauchen keinen Dialog der Religionen (oder Kulturen), wir brauchen Solidarität zwischen denen, die in moslemischen Ländern für Gerechtigkeit kämpfen, und denen, die an den gleichen Kämpfen anderswo teilnehmen.“ (Žižek 2013, 107)

Die liberale Lebenshaltung wird zur generellen Lebenshaltung eines lizensierten Genießens: Kaffee ohne Koffein, Bier ohne Alkohol, Cola, aber nur Diät-Cola, Sex wird befürwortet als Beitrag zur Gesundheit, Zigaretten als E-Zigarette, die Revolution darf nicht nach Revolution riechen. Žižek führt dieses Argument sehr oft an, es besagt, dass der Liberalismus die Radikalität der Begriffe von innen her zügelt und das Genießen begrenzt - aber er diskutiert nicht den rationalen Gehalt und verbindet die Kritik nicht mit alternativen Lebensformen: wäre es denn richtig zu rauchen oder exzessiv Alkohol zu trinken, nur weil der Liberalismus sich dem entgegenstellt? Der Geist von 68 wird aufgenommen, das Recht auf Abtreibung, die Homo-Ehe, das erkläre das Geheimnis der Ruhe, die in den vergangenen vierzig Jahren herrsche (Žižek 2011, 267). Der politisch korrekten, kulturalisierten Linken bleibt der Kampf gegen den Totalitarismus und Fundamentalismus, eigene, entschiedene Ziele darf sie nicht verfolgen, wenn sie nicht autoritär und totalitär sein will. So verstrickt sie sich in spezifische Widersprüche: der pazifistisch-humanistische Anspruch auf die Verteidigung der Menschenrechte schlägt um in die Nutzung militärischer Mittel und Folter, dieselben Leute, die für die Legalisierung von Drogen sind, unterstützen ein Rauchverbot, Mitglieder fremder Kulturen dürfen nicht für das kritisiert werden, was in der eigenen Gesellschaft abgelehnt wird (Gewalt gegen Frauen, autoritäre Erziehung). Es geht um einen „verallgemeinerten multikulturalistischen Historismus“ (2009, 61). 
Der humanistische Liberalismus verwickelt in die Widersprüche, weil er seinem Prinzip nach keine „ungeschriebenen Regeln“, keine gesellschaftliche Substanz mehr für das hat, was Žižek als „Anstand“ und als „Höflichkeit“ bezeichnet. Er argumentiert hier quasi-hegelianisch, wonach die „unerbittliche Logik des Kapitals“(Žižek 1998, 95), die digitale Kontrolle über das Leben, die Biogenetik oder ökologische Herausforderungen die selbstverständlichen „sittlichen“ Grundlagen, die soziale Substanz freier, unabhängiger Individuen und ihrer Solidarität miteinander untergraben. Diese Prozesse erscheinen Žižek (2011,302ff) als bedrohlich, weil sie den großen Anderen (die symbolische Substanz), also den Realitätssinn selbst, die Grundkoordinaten unserer Erfahrung erschüttern. Mehr denn je sei das heutige Subjekt von einem unerbittlichen Zwang betroffen, der sein Leben antreibt. Jene Prozesse werden von Žižek als solche der Proletarisierung bezeichnet und sollen auf einen apokalyptischen Punkt verweisen. Tatsächlich lebten wir auch in apokalyptischen Zeiten. Demgegenüber sei es ein Zeichen des moralischen Fortschritts, wenn dogmatisch klar sei, dass Gewalt gegen Frauen und Vergewaltigungen nicht akzeptabel seien, dass es Redefreiheit gebe, Achtung individueller Freiheiten auf Kosten von Gruppenrechten, die Freiheit, alles und jeden öffentlich anzugreifen. Wenn nun die Folter von Individuen durch US-Regierungsinstanzen offen zugestanden würde, dann würde nicht einfach etwas offen zugestanden, alle wüssten ohnehin, dass staatlicherseits gefoltert würde. Mit diesem Sprechakt der Offenlegung würden wir jedoch alle zu Opfern: „Wir sollten uns darüber im Klaren sein, dass dadurch ein wertvoller Teil unserer kollektiven Identität unwiederbringlich verloren ist. Wir befinden uns mitten in einem Prozess des moralischen Verfalls. Die Machthabenden versuchen buchstäblich, einen Teil unseres ethischen Rückgrats zu brechen und die wohl größte Errungenschaft der Zivilisation - die Zunahme unseres spontanen moralischen Empfindens - zu schwächen und zunichte zu machen (vgl.Žižek 2009, 85, 86f, 120). Žižek plädiert hier dafür, an westlichen Traditionen und Werten festzuhalten, also an dem in der westlichen Moderne verankerten Universalismus. Der Liberalismus sei jedoch nicht stark genug, seine eigenen Grundwerte wie Freiheit oder Gleichheit vor einem fundamentalistischen Angriff zu schützen, weil er diesen selbst generiert. Žižek bezieht sich angesichts des rechten Populismus oder islamistischer Bestrebungen mehrfach aufWalter Benjamins Aussage, wonach der Faschismus für eine gescheiterte Revolution stehe. Allein eine erneuerte Linke könne das Erbe des Liberalismus retten (vgl. Žižek 2011, 288f). Er wendet sich damit gegen diejenigen, die das als kulturimperialistisch ablehnt - für ihn ein weiteres Zeichen, die Geschichte zu leugnen und die konkrete Form des Universalismus zu verwerfen, der in der lokalen Kultur Europas seine Wurzeln habe. Die gegenwärtige Ideologie ziele nicht mehr auf die Anrufung der einzelnen, um sie dadurch zu Subjekten zu machen - wie Althusser oder Foucault vermutet haben -, vielmehr seien die 
symbolischen Eigenschaften temporär und flexibel, „weswegen das Individuum ständig dazu aufgerufen ist, sich » neu zu erschaffen «" (ebd., 62). Aus dem Blinkwinkel des Liberalismus erscheinen Verhältnisse oder Identitäten als kontingent, als konstruiert und Ergebnis eigener Leistung. Mit ihrer Auffassung, dass die sozial-ideologischen Einheiten Produkt von kontingenten diskursiven Kämpfen darstellten, fügen sich aus Žižeks ideologiekritischer Sicht auch Butler oder Laclau in die liberale Ideologie ein. Der Schwerpunkt würde vom antikapitalistischen Kampf auf die vielfältigen Formen des politisch-ideologischen Kampfes um Hegemonie verschoben. „Alle authentischen historischen Spannungen [würden] in die endlosen performativen Spiele einer ewigen Gegenwart auf[gelöst]" (Žižek 2009, 19f, 132; Žižek 2011,273). Auf diese Weise enthistorisiert der Liberalismus die gesellschaftlichen Prozesse und bindet auch emanzipatorische Theorien in den Prozess der permanenten Selbstrevolutionierung des Kapitalismus ein.

Für den Liberalismus ist das Ende der Geschichte erreicht. Die Zeit der großen Erzählungen, Ideologien, Erklärungen, Fundierungstheorien soll vorbei sein. Allein ein „schwaches“ Denken würde aus liberaler Sicht der nomadischen und rhizomatischen Textur der Realität gerecht. Die Gesellschaft werde in eine Vielzahl von Identitäten und Interessen aufgelöst, die in ihrer Differenz nebeneinander stehen und in ein Verhältnis wechselseitiger Toleranz eintreten. Žižek demonstriert, wie sehr sich kritische Begriffe ins liberale Dispositiv eingliedern lassen und bestreitet jene Diagnose. Was Badiou, Laclau oder Butler fehle, sei eine Metatheorie der Geschichte, die auf die Frage eine Antwort geben könne, ob der Übergang vom Essenzialismus zur Kontingenz, von der großen zur lokalen Geschichte und Politik eine Einsicht in historische Prozesse und mögliches politisches Handeln sei oder ideologische Täuschung. „Eine solche Metatheorie kann nur der historische Materialismus liefern, der politische Prozesse in ihren globalen sozioökonomischen Kontext einordnet (Žižek 2009, 212). Žižek sucht nach dem dritten Punkt, von dem aus das „zugrundeliegende Strukturprinzip“ angegriffen werden kann, das die falschen Gegensätze in Spiel bringt (ebd., 93; 2011, 288).

Der Liberalismus entpolitisiert, er transformiert gesellschaftliche Antagonismen in Differenzen zwischen sich tolerierenden Gruppen. Auf dieser Grundlage kehrt das Verdrängte des grundlegenden Konflikts der Gesellschaft als verschobener und traumatischer Gegensatz zurück: anstatt Klassenkampf, der sich aufgrund innergesellschaftlicher Gegensätze bildet, wird eine integrale Totalität unterstellt, die an den Rändern von außen bedroht wird: die Juden, der islamistische Terror, die MigrantInnen. Der Antisemitismus sei die Ideologie an sich: der soziale Widerspruch wird mystifiziert und seine Ursache auf den äußeren Eindringling projiziert (Žižek 2013, 39). Die Konstruktion eines universalistischen, die Individuen verpflichtenden Projekts soll vermieden werden. Žižek (1998, 33ff) unterscheidet mehrere Formen der Entpolitisierung, die den 
politischen Konflikt leugnen. a) In der Arche-Politik wird kommunitaristisch versucht, einen organisch strukturierten, homogenen gesellschaftlichen Raum zu definieren, der keine Möglichkeit für das politische Ereignis einräumt. b) In der Parapolitik wird die Politik aus dem Antagonismus herausgeführt und in Polizeilogik übersetzt, der politische Konflikt verwandelt sich in einen Wettkampf zwischen anerkannten Parteien. Für diese Konzeption stehen Rawls oder Habermas. c) In der marxistischen Meta-Politik gilt der politische Konflikt lediglich als ein Ereignis, das auf der Ebene der Ökonomie seinen eigentlichen Platz hat. d) In der Ultra-Politik wird der Konflikt bis zum Extrem der militärischen Auseinandersetzung gesteigert. Es gebe hier keinen gemeinsamen symbolischen Raum mehr. e) Zeitdiagnostisch hält Žižek eine fünfte Form der Verleugnung des Politischen für entscheidend: die Postpolitik. Sie entspricht dem liberalen Multikulturalismus, denn in diesem Fall wird der Konflikt zwischen globalen ideologischen Visionen durch die Kollaboration von aufgeklärten Technokraten und liberalen Multikulturalisten ersetzt, die sich mittels Aushandlung um mehr oder weniger universalen Konsens bemühen, der auf eine pragmatische rationale Politik der neuen, radikalen Mitte zielt, also für das Projekt Tony Blairs und New Labour. Diese besondere Negation der Politik durch Clinton und die Sozialdemokratie des Dritten Weges in Westeuropa trug Žižek $(2009,134)$ zufolge zur „einschneidensten Transformation“ bei, die der Kapitalismus erfahren habe. Erst indem Blair den Thatcherismus wiederbolte und in neuen institutionellen Formen verankerte, erhob er die Zufälligkeit zur Notwendigkeit. So war die sozialdemokratische Postpolitik eine „echte Option“ für den Kapitalismus.

Dies wirft für Žižek die besondere Frage auf, wie überhaupt der Kapitalismus selbst überwunden werden kann, wenn dieser sich selbst ständig revolutioniert und noch das, was als Hindernis (wohlfahrtsstaatliche Sicherheiten, regulatorische Eingriffe, Proteste der sozialen Bewegung) erscheint, schließlich zu seiner Selbsttransformation und Beschleunigung nutzen kann (vgl. Žižek 2002, 138). Vielleicht schreibt Žižek deswegen etwas rätselhaft, dass in „gewisser Weise der Kapitalismus unzerstörbar" sei (Žižek 2009, 132). Die Konsequenzen dieser lapidaren Äußerung sind ja erheblich; und tatsächlich beschäftigt das Problem Žižek dann auch in späteren Texten. Zu Benjamins Vorschlag, die Revolution als Griff nach der Notbremse zu verstehen, die den Zug anhalten könnte, äußert er sich einmal eher skeptisch, um dann doch - wie auch schon Claus Offe in den 1980er Jahren - in dieser Empfehlung die Lösung zu sehen. Der Feind sei nicht mehr der Staat - aber für wen in der Linken ist das Fall?, möchte man zurückfragen -, sondern der Fluss der permanenten Selbstrevolutionierung, der von einem symptomalen Torsionspunkt aus zu unterminieren sei (vgl. Žižek 2009, 291; Žižek 2011, 311, 326). Entsprechend empfiehlt er, es müsse ein dritter Weg gesucht werden zwischen der institutionalisierten parlamentarischen Politik und den neuen sozialen 
Bewegungen (Žižek 2002, 138). Wie auch schon Nicos Poulantzas in Staatstheorie schlägt er vor, über die falsche Wahl hinauszugelangen: entweder den Staat zu übernehmen oder zu ihm auf Distanz zu gehen, sondern ihn, seine Funktionsweise, sein Verhältnis zur Ökonomie zu transformieren. Die Frage, die er sich angesichts der Proteste des Jahres 2011 stellt, ist: was in den Tagen danach passieren soll. Sie hätten das Tabu gebrochen, über Alternativen dürfe wieder nachgedacht werden. Sei vorher der Klassenkampf-Essenzialismus zugunsten der Pluralität antirassistischer, feministischer und anderer Kämpfe aufgegeben worden, werde nun wieder „Kapitalismus“ als Begriff für das entscheidende Problem verstanden. Denn auch wenn einmal Demokratie durchgesetzt sei, gibt es immer noch Armut. Das Problem der bestimmten Negation kehre für die heutige Linke also zurück: „Welche neue positive Ordnung sollte die alte ersetzen, wenn die erhabene Begeisterung für den Aufstand erst einmal verschwunden ist?" Die Proteste brächten eine authentische Wut zum Ausdruck, aber blieben unfähig, sich in ein „minimales positives Programm eines gesellschaftlich-politischen Wandels zu transformieren. Sie bringen einen Geist der Revolte ohne Revolution zum Ausdruck.“ (Žižek 2013, 119) Der Erfolg sei nicht an der erhabenen Ehrfurcht vor den ekstatischen Momenten des Protests, der Revolte oder Revolution zu bemessen, sondern ,an den Veränderungen, die das große Ereignis am Tag danach im alltäglichen Leben hinterläßt“ (Žižek 2011,331). Genau an diesem Punkt seien auch die Französische und die Russische Revolution gescheitert. Dass es sich heute so verhält, dass der Widerstand gegen das System keine realistische Alternative formuliere, sei eine ,schwerwiegende Anklage gegen unsere Epoche“. Denn die weltlose Ideologie des Kapitalismus bringe die Menschen um jegliche bedeutungsvolle Ideologie (ebd., 87).

Žižek wendet sich gegen die populistische Versuchung, ,unsere Wut“ auf impotente Weise auszuleben, sondern sie in kalte Entschlossenheit zu verwandeln (vgl. Žižek 2009, 23; vgl. hingegen die sozialdemokratische Haltung bei Streeck 2013). Vor diesem Hintergrund schließt er an Rancières Überlegung an. Es geht darum, eine neue Politik durchzusetzen, durch die diejenigen, die in der bestehenden Gesellschaft gar nicht vorkommen, die keinen Anteil haben, die politischen Koordinaten verschieben und sich zur Geltung bringen. „Was wir bräuchten, wäre ein echter Akt: eine symbolische Intervention, die den großen Anderen (die hegemoniale soziale Verbindung) unterminiert und dessen Koordinaten neu bestimmt." (Žižek 2009, 90) Emanzipatorische Politik würde die Mannigfaltigkeit derer, die keinen Platz in der Gesellschaft haben, auf eine minimale Differenz „Zwischen dem herrschenden allgemeinen gesellschaftlichen Prinzip und denen, die durch ihre Existenz dieses Prinzip unterminieren“" reduzieren (ebd., 225). Es stellt sich die Frage, in welchem Sinn hier von Klassenkampf gesprochen werden kann und ob Žižek nicht doch dazu tendiert, einen Widerspruch eher an den Rändern der Gesellschaft zu konstruieren. In dieser Konzeption ist Klassenkampf 
nicht dualistisch und getragen von zwei reinen Klassen, die sich gegenüberstehen. Vielmehr richtet er sich aufgrund eines dritten Elements gegen den aufgezwungenen Hauptwiderspruch. Bei diesem dritten Element handelt es sich um einen unteilbaren und verworfenen Rest (zum Beispiel im Nationalsozialismus der „Jude“). Dieser Rest verleugnet fetischistisch den Klassenantagonismus, steht aber gerade deswegen für ihn und verhindert den Klassenfrieden. Die Klassen können keine positive Identität gewinnen und sich harmonisch ergänzen. „Gerade weil es niemals nur zwei gegensätzliche Klassen gibt, gibt es Klassenkampf.“ (Ebd., 186; vgl. aber Žižek 2011, 278ff, wo er deutlich macht, wie gefährlich das Argument werden kann, wenn Linke sich wegen deren Antiimperialismus auf islamistische Bewegungen mit ihrer gegenaufklärerischen und antiuniversalistischen Orientierung beziehen und nicht noch zusätzliche emanzipatorische Kriterien anwenden.) Es ist dieser verworfene Rest, der bei aller Partikularität als Teil, der keinen Anteil hat, die Universalität selbst verkörpert: der das Singuläre direkt mit dem Allgemeinen verbindet, eine Gemeinde der Gläubigen, die Weltbürgergesellschaft ist. Denn von dort her reorganisiert sich das Ganze und gibt allen Elementen eine neue Bedeutung: nur noch Kämpfer für die Emanzipation auf der einen Seite, reaktionäre Gegner auf der anderen Seite (vgl. 2011, 250).

Slavoj Žižeks Texte bieten ein besonderes Modell der kritischen Gesellschaftsanalyse, das nahelegt, zahlreiche Elemente einer materialistischen Analyse von Kräfteverhältnissen mit Zeitdiagnose zu verbinden. Die Analysen bewegen sich auf einer besonderen Ebene, auf der der Kapitalismus eher als Repräsentation gefasst werden kann und etwas von einem Traum hat. Wesentlich für Žižeks Analysen sind auch die Stellen von Marx, in denen er die besondere semiotische Bedeutung von Louis Bonaparte für die verschiedenen sozialen Klassen Frankreichs untersucht. Ansonsten bleiben Žižeks Beschreibungen des Kapitalismus oberflächlich und eher thetisch, ohne dass Zusammenhänge diskutiert würden. Auch wenn er von sozialen Klassen oder gar dem Proletariat spricht, handelt es sich eher um Metaphern, mit denen spezifische symbolische Prozesse bestimmt werden. Den inneren Zusammenhang der bürgerlichen Gesellschaft, die Bewegung ihrer Widersprüche, um den es Adorno geht, bekommt er nicht in den Blick; ebensowenig konkrete soziale Kräfte, so dass erklärt würde, warum eine besondere Gruppe von Menschen auf diese oder jene Weise handeln, in bestimmte Konflikte geraten und die gesellschaftliche Entwicklung festlegen. Die kulturellen Prozesse, die intellektuellen Praktiken - auch dessen, was Gramsci als Philosophie der Praxis bezeichnet - werden von Žižek gar nicht näher untersucht. Es ist eine Eigenart seines Ansatzes, die besondere Wirksamkeit von Ideologie in den Blick zu nehmen, genau genommen den Kapitalismus selbst als eine Ideologie. Dabei geht es darum, durch Ideologiekritik Mechanismen frei zu legen, die linkes Denken blockieren und den Raum für die Kritik der politischen Ökonomie zu 
schaffen. Diese selbst ist aber nicht sein Gegenstand, sondern die innerideologischen Kämpfe um das Verhältnis der Linken zur Wirklichkeit und der Glaube an ihren Glauben, die Verhältnisse ändern zu können. Die vielen christlichen Anspielungen sind also nicht von ungefähr. Die Einwirkung auf das Verhältnis der Linken zur Wirklichkeit soll bewirken, dass die Linke sich nicht in falsche Widersprüche verwickelt, an falsche Identitäten binden lässt, dass sie nicht in die Falle von fragwürdigen Begriffen wie „Totalitarismus“ oder Geschichtskonstruktionen geht. Sie soll nicht naiv sein und wahrnehmen, dass ihre eigenen Theorien einen Funktionswechsel erfahren können, der zur Herrschaft beiträgt. Es geht darum, sich vom großen Anderen frei zu machen und von der Furcht, Furcht zu haben, um die Fähigkeiten, den Willen zum Handeln und öffentlichen Vernunftgebrauch auszubilden. Dies meint, eine Verbindung zwischen dem Singulären und dem Universellen direkt herzustellen. Daraus resultieren jedoch ein eigener Voluntarismus und eine oftmals problematische gewalthafte Rhetorik, die nahelegt, dass wir uns heute zwischen Apokalypse und Revolution bewegen. Dieser Voluntarismus steht in einem unaufgeklärten Missverhältnis zu den Bezugnahmen auf ethische und liberale Begriffe der Freiheit, der Gleichheit, der Solidarität und der Demokratie auf der einen Seite und marxistischen Begriffen der Analyse konkreter Verhältnisse auf der anderen.

\section{Literatur}

Adorno, Theodor W. (1951): Minima Moralia, in: ders., Gesammelte Schriften, Bd.4, Frankfurt/M. 1980.

- (1964): Philosophische Elemente einer Theorie der Gesellschaft, in: ders., Nachgelassene Schriften

IV.12, Frankfurt/M. 2008.

- (1966): Gesellschaft, in: ders., Gesammelte Schriften, Bd. 8, Frankfurt/M. 1972.

- (1968): Einleitung in die Soziologie, in: ders., Nachgelassene Schriften IV.15, Frankfurt/M. 1993. Althusser, Louis (2010): Ideologie und ideologische Staatsapparate, in: ders., Gesammelte Schriften, hrsg. von Frieder Otto Wolf, Hamburg.

Badiou, Alain, Žižek, Slavoj (Hrsg.) (2012): Die Idee des Kommunismus, Bd. II, Hamburg. Brown, Wendy (2000): Reflexionen im Zeitalter der Identität, in: Rainer Forst (Hg.): Toleranz. Philosophische Grundlagen und gesellschaftliche Praxis einer umstrittenen Tugend, Frankufurt/ New York.

Douzinas, Costas, Žižek, Slavoj (Hrsg.)(2012): Die Idee des Kommunismus, Bd. I, Hamburg. Gramsci, Antonio (1996): Gefängnishefte, Bd. 7, Hamburg.

Poulantzas, Nicos (1974): Politische Macht und soziale Klassen, Frankfurt/M.

Streeck, Wolfgang (2013): Gekaufte Zeit, Frankfurt/M.

Žižek, Slavoj (1998): Ein Plädoyer für Intoleranz, Wien.

- (2002): Die Revolution steht bevor. Dreizehn Vesuche über Lenin, Frankfurt/M.

- (2009): Aufverlorenem Posten, Frankfurt/M.

- (2011): Die bösen Geister des himmlischen Bereichs. Der linke Kampf um das 21. Jabrhundert,

Frankfurt/M.

- (2013): Das Jahr der gefäbrlichen Träume, Frankfurt/M. 